\title{
Higher order reversal curves in some hysteresis models
}

\author{
KrzYsztof ChWASTEK \\ Faculty of Electrical Engineering, Częstochowa University of Technology \\ Armii Krajowej 17, 42-200 Częstochowa, Poland \\ e-mail:krzych@el.pcz.czest.pl
}

(Received: 25.01.2012, revised: 14.03.2012)

\begin{abstract}
Some physical concepts important for a hysteresis model (effective field, anhysteretic magnetization) are discussed on the example of Jiles-Atherton model. The Jiles-Atherton model reveals some drawbacks, which make this model more difficult to be applied in electrical engineering. In particular, it does not describe accurately the magnetization curves after a reversal, moreover complex magnetization cycles are poorly represented. On the other hand, the phenomenological description proposed by Takács seems to be a valuable alternative to the Jiles-Atherton formalism. The concept of effective field may be easily incorporated in the description.
\end{abstract}

Key words: soft magnetic materials, hysteresis, modelling

\section{Introduction}

Hysteresis is prevalent in physics, biology and economics, therefore the descriptions of the phenomenon attract the attention of many scientists. Ferromagnetic hysteresis is important for physicists, designers of magnetic circuits in electric machines and power system engineers. Physicists and materials science engineers are interested mainly in understanding the physical properties of the material and their relation to microstructure [9, 12, 38, 46, 75-77]. The research scope for the designers of electrical machines covers macroscopic descriptions of phenomena occurring within a ferromagnetic core and the possibility to include them in circuit simulations or finite element/volume codes in order to optimise the structure and properties of developed devices $[5,36,37,41,59,69,79]$. Finally, power system engineers strive to develop simplified yet realistic hysteresis models useful for prediction of system behaviour during inrush, ferroresonance and in other transient states $[15,16,33,39,62,67,78,80]$.

The present paper focuses on some aspects of hysteresis modelling, important for electrical engineers. The impact of distorted flux density waveform on power loss and the possible approaches to model this effect shall be the subject of another paper. The concepts how to predict an arbitrary magnetization curve on the basis of knowledge of the major (saturating) hysteresis loop are discussed on the example of macroscopic phenomenological description 
advanced by Jiles and Atherton [46], due to its extreme popularity in the scientific community, and on the example of one of the most promising models available nowadays, proposed by Takács $[73,74]$. Yet, the presented concepts may be (or have already been) relatively easy adapted to other phenomenological hysteresis models. The necessity to take into account the rate-dependence of magnetization curves is pronounced. Some higher order reversal curves including rate-dependent effects are modelled using a recent dynamic extension to the Takács description [26].

\section{Jiles-Atherton model}

The Jiles-Atherton (J-A) model [46] still remains one of the most widely used macroscopic hysteresis descriptions nowadays. It is based on the concept that hysteresis is the result of impedances to changes of magnetization occurring in the bulk ferromagnetic material. The source of impedances are the defects in the solid such as nonmagnetic inclusions, voids or regions of inhomogenous stress. The fundamental model equation is an ordinary differential equation, where the independent variable is the so-called effective field, being the true field within the material, $H_{\text {eff }}=H+\alpha M$. The concept of effective field makes it possible to describe the cooperative action between magnetic moments within the magnetic core material. The Jiles-Atherton relationship may be written

$$
\frac{d M_{i r r}}{d H_{\text {eff }}}=\frac{M_{a n}-M_{i r r}}{k \delta},
$$

where $M_{i r r}$ denotes the irreversible component of magnetization, whereas $M_{a n}$ is the anhysteretic magnetization, which corresponds to the state of global equillibrium. The aforementioned equation describes the evolution of the irreversible magnetization component and needs to be supplemented with appropriate formulas describing the anhysteretic, sigmoid-like curve and the relationship between the irreversible and total magnetization. Originally the model developers have used the modified Langevin equation for the anhysteretic curve and a weighting coefficient $c$. Chwastek has proposed to use the modified Brillouin function instead of the Langevin function (what is physically judged) and modified the relationship between the total and the irreversible magnetization [23, 24], making its structure similar to that one proposed by Kádár for the product Preisach model [52-54]. The latter modification shall be discussed in greater detail in the consecutive part of the manuscript.

Figure 1 presents an exemplary major hysteresis loop and anhysteretic curve, obtained after solution of the J-A equations for an arbitrary set of model parameters. The model equations have been solved for the inverse excitation case following the guidelines presented in $[22,69]$. The set of model parameters used in the simulation was $\alpha=10^{-3}[-], a=k=2000[\mathrm{~A} / \mathrm{m}]$, $c=0,1[-], M_{s}=1.710^{6}[\mathrm{~A} / \mathrm{m}]$. It was chosen to be similar to parameter sets used by model developer in his papers on parameter estimation $[48,49]$. The maximum flux density was $B_{m}=1,5[\mathrm{~T}] . \delta$ denotes the sign of derivative $d M / d t$, whereas $\delta_{M}$ is introduced to avoid negative susceptibilities after field reversal (it will be discussed in detail in the consecutive part of the paper). 


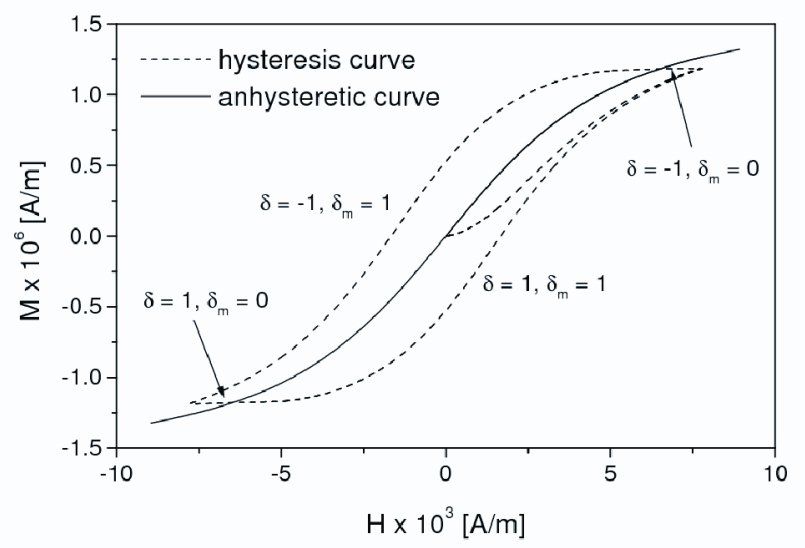

Fig. 1. Major hysteresis loop and anhysteretic curve in the Jiles-Atherton model

\section{Minor loops, reversal curves and Madelung rules}

Let us consider an arbitrary time variation of magnetization $M(t)$ and field strength $H(t)$ as depicted in Figure 2, which presents the corresponding M-H plane. As reversal point we shall understand any time instant, when the control variable changes its sign. Thus points B, C, D and $\mathrm{E}$ are reversal points (sometimes referred to as turning points [34].

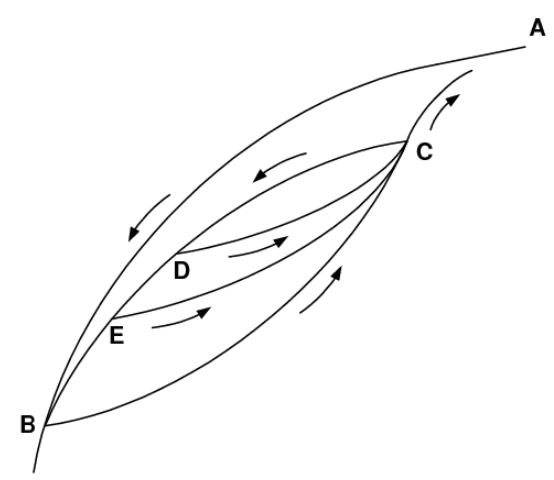

Fig. 2. An illustration of minor loops, reversal points and Madelung rules

Any phenomenological hysteresis model acceptable for engineering applications should be able to represent accurately not only the shape of major loop (limiting cycle curve, which is most often sigmoid-like), but also it should be useful for prediction of more complicated magnetization cycles. The major loop is sometimes referred to as the Zero Order curve. Let us assume that the segment between points A and B belongs to the major hysteresis loop. At point $B$ the sign of control variable changes and a First Order Reversal Curve begins (denoted as B-C). By analogy, the segments C-B, C-D and C-E are Second Order Reversal Curves, whereas E-C and D-C are Third Order Reversal Curves and so on. 
The regularity of shapes of magnetization curves has been noticed over a century ago and expressed using Madelung rules [60], cited later in [37, 45, 64, 83, 84]. The rules may be expressed as:

1) All first-order reversal curves starting from major loop and going to saturation (like B-C curve in Fig. 2) are determined only by their starting point (here B) (major loop returnpoint memory); otherwise the part of any reversal is determined by the whole history of magnetization.

2) If some point $\mathrm{C}$ of any reversal curve starting from reversal point $\mathrm{B}$ becomes a new reversal point, then the curve C-E-B returns to the initial point B (minor loop return-point memory).

3) If the point $\mathrm{E}$ of the curve $\mathrm{C}-\mathrm{E}-\mathrm{B}$ becomes the newest reversal point and the transition curve $\mathrm{E}-\mathrm{B}$ extends beyond the point $\mathrm{C}$, it will pass along the section $\mathrm{C}-\mathrm{A}$, as if the loop C-E-C did not exist at all (wiping out property).

The scientific community generally accepts these rules, as confirmed by many precise experiments.

Many physicists have noticed the existence of power laws occurring between different parameters of minor loops in dependence on maximum flux density (or magnetization) [42, 57, 75-77]. This idea might have been inspired by the works of Steinmetz [71], who examined the dependence of power loss on maximum flux density. The aforementioned power laws may become reflected in power laws concerning the values of some model parameters, in particular those related to average domain size [23, 24]. In the case of the Jiles-Atherton model, the appropriate parameters are $a$ and $k$. The "classical" Jiles-Atherton model, which does not update any of its parameters for more complicated remagnetization cycles, does not represent the shapes of minor loops well, as pointed out in a number of references e.g. [10,58].

In 1992 Jiles has presented an extension of his model [47], based on the "volume fraction" concept [55], borrowed from the Preisach model. The idea relies on the assumption, that for a given minor loop (say B-C-B in Fig. 2), the knowledge of minor loop tip coordinates allows one to introduce a correction factor for the equation for differential susceptibility $\mathrm{dM} / \mathrm{dH}$, valid for that minor loop "excursion". In the context of the Preisach model, volume fraction means that only a certain part of "hysterons" contributing to the total hysteresis loop gets involved in the remagnetization process [61], cf. Figure 3.

In [23] this conjecture has been transformed into a practical Jiles-Atherton model extension for modelling minor loops, including asymmetric ones, by the introduction of the "effective" magnetization amplitude, defined as

$$
M_{\text {minor }}=0,5\left|M_{\mathrm{TIP} 1}-M_{T I P 2}\right|,
$$

where $M_{\mathrm{TIP} 1}$ and $M_{\mathrm{TIP} 2}$ denote magnetization values for both loop tips. Update of values of $a$ and $k$ parameters using the above-given value of the "effective" magnetization referred to saturation magnetization as the argument of power laws made it possible to improve the representation of minor loops.

It has to be admitted, that despite the aforementioned modification of some values of the Jiles-Atherton model parameters is physically judged, it cannot be applied directly to system 
studies in power engineering, when the knowledge of extreme magnetization values during transient states is usually unknown a priori. In the author's opinion this fact greatly limits the application scope of the Jiles-Atherton description in electric power engineering. Yet the literature reports on some such attempts $[15,16,80]$. On the other hand, it seems that quite satisfactory modelling results may be obtained using for this purpose simple polynomial-based descriptions $[33,67]$.

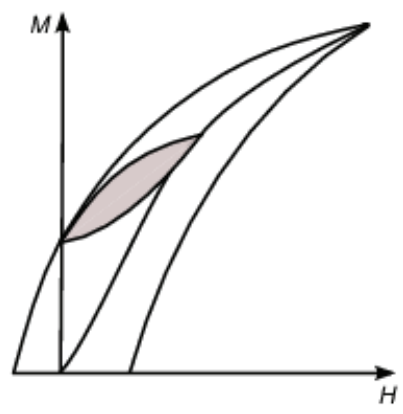

Fig. 3. An illustration of volume fraction concept

Another possible approach to the problem how to modify the values of J-A model parameters has been advanced in $[13,58]$. At first the equation, which describes one of the branches of the major loop is solved in the $M-H_{\text {eff }}$ space. Then a scaling factor is introduced, which makes it possible to predict the reversal curves corresponding to the same sign of derivative $d M / d t$. This method is similar in spirit to the approach proposed in [83, 84], but there are still some problems related to the introduction of reversible processes into the description.

One of the key achievements of Jiles and Atherton is noticing the fact that remagnetization does not only consist of irreversible, but also reversible processes. However the way they have taken reversibility into account has blurred the forthcoming model extensions. In fact the parameter $c$ given as the ratio of the slopes $d M / d H$ to $d M_{a n} / d H$ at loop origin [49] is incorrectly defined, because the anhysteretic curve has singularity at that very point. Taylor expansion of the function may be really helpful, but the iterative procedure proposed in the paper on estimation of model parameters [49] contradicts the concept, that some of model parameters need to be updated. Solution sets of J-A model parameters may be ambiguous $[50,51,65]$ or far from optimal ones [65]. Other problems with the classical estimation method have been discussed previously [21]. It is believed that the use of redundant number of data points makes it possible to partially remove some problems with data noise [19]. The choice of an alternative robust estimation method, either an AI stochastics-based $[5,56,58]$ or a deterministic one [19] is up to the potential model user.

The problem with the correct inclusion of reversibility in the J-A description does not only affect the values of model parameters, but also reflects itself in a poor representation of reversal curves and minor loops. Of particular interest is the occurrence of the regions of negative susceptibility obtained for lower excitation level after a change of $d M / d t$ sign $[10,49,58,79]$. This phenomenon may affect the numerical solution of the FEM code 
including the J-A engine. In order to avoid this problem, an additional pseudo-variable $\delta_{M}=$ $=0,5\left[1+\operatorname{sign}\left(\left(\mathrm{M}_{\mathrm{an}}-M\right) d M / d t\right)\right]$ may be introduced [30, 19]. This term suppresses the irreversible term until magnetization crosses the $M_{a n}\left(H_{e f f}\right)$ curve. Its existence may be justified by the words: "In general, the slope of the $\mathrm{M}-\mathrm{H}$ curve is different before and after a turning point. The reason for the difference in slope is that before the turning point, the change in magnetization is due to both the reversible and the irreversible processes. After the turning point, only the reversible process contributes. This is due to the fact that the reversible process does not return any energy to the applied field; hence $d M / d H$ is zero for this process" [34].

In fact, switching off the $d M_{i \mathrm{rr}} / d H$ component after a turning point using $\delta_{M}$ term is a brute-force approach and only partially removes the peculiar model behaviour, due to the coupling of both irreversible and reversible processes in the effective field, which in turn affects the instant value of anhysteretic magnetization. In some Preisach-type models, both magnetization components may be totally decoupled, this concept is called State-Independent Hypothesis [14]. Experiments indicate that the initial slope of the reversal curve in quasi-static conditions should rather be non-zero, close to the value of initial permeability obtained from the Rayleigh equation. In dynamic conditions, the situation becomes much more complicated due to smearing of the loop tips caused by eddy currents.

The problem with zero initial permeability for the classical Preisach model has been patched by the introduction of the moving and the product extensions [34, 35]. Following the idea of product model by Kádár $[52,54]$ Chwastek has introduced a modulation of total susceptibility with a magnetization-dependent term $R(m)[23,24]$

$$
\frac{d M}{d H}=R(m)\left[\beta+\frac{d M_{i r r}}{d H}\right],
$$

where in the first approximation $R(m)=1-\left(M / M_{s}\right)^{2} . \beta$ denotes the initial (reversible) permeability expressed in relative units. For the Rayleigh range the magnetization process is assumed to be practically reversible. The consecutive extension of the product model, which addresses the existence of different anisotropy classes in magnetic materials, affecting the form of $R(m)$ function [53, 54] has been followed in [27, 29]. It should be noted that the introduction of $R(m)$ term makes it possible to correct the susceptibilities of minor loops thus addressing the so-called non-congruency issue [61]. The experimentally determined dependences of the reversible susceptibility versus total magnetization obtained from small field reversals are qualitatively close to a parabolic profile of the $R(m)$ function [4].

As pointed out earlier, the concept of effective field adapted from [81] plays a fundamental role in the J-A description. It is also important to notice the paramount role of the effective field in any attempts to develop hysteresis models compliant with thermodynamics of irreversible processes [6, 7]. It has been shown that the introduction of the positive feedback mechanism expressed by the effective field either improves the overall accuracy of other hysteresis models or constitutes their foundations $[28,35,44,61,70]$. The effective field term in the J-A model has been extended to take into account a number of phenomena affecting the shape of hysteresis loop, e.g. magnetostriction [68] or eddy currents [72]. 


\section{Takács model foundations}

The Takács model is based on a hyperbolic tangent transformation of loop coordinates. The author uses dimensionless variables for brevity of notation in his papers [73, 74]. The variable at the abscissa axis has been identified as the effective field expressed in relative units, whereas the variable at the ordinate axis as total magnetization, also in relative units [26]. This assumption makes it possible to introduce a depiction of hysteresis as a self-sustainable relationship $M=\hat{\Gamma}\left(H_{\text {eff }}\right)$ or $H_{\text {eff }}=\hat{\Gamma}^{-1}(M)$, where $\hat{\Gamma}$ is the hysteresis operator [3, 26].

In dimensionless form the basic model equations for a symmetrical loop may be written as

$$
\begin{gathered}
f_{+}=\tanh \left(x-a_{0}\right)+b_{0} \text { for the ascending branch, } \\
f_{-}=\tanh \left(x+a_{0}\right)-b_{0} \text { for the descending branch, } \\
b_{0}=0.5\left[\tanh \left(x+a_{0}\right)-\tanh \left(x-a_{0}\right)\right] .
\end{gathered}
$$

In the abovegiven description the reversible term (assumed by model developer as a linear function of $x$ ) is skipped for the sake of simplicity. It does not affect the basic concept of the model, but its presence highly complicates the identification method. The meaning of parameter $a_{0}$ is the normalized coercive field strength.

It is important to capture the meaning of $b_{0}$ and similar constants defined for successive reversal points. The purpose of $b_{0}$ is to assure that both loop branches coincide at a reversal point (in other words the loop tip should lie on the anhysteretic curve). Tracking the coordinates of reversal points (i.e. their storage and retrieval using the stack concept [55] makes it possible to develop a global memory mechanism, similar to the one implemented in the Preisach model [61]. For a symmetrical loop with sufficiently high amplitude (major or saturating loop) the term $b_{0}$ approaches zero.

The model developer has presented in his papers and books a number of practical formulas, useful for description of different magnetization conditions and phenomena. Let us refer again to Figure 2. If the section $\mathrm{AB}$ belongs to the major loop (zero order curve), the section $\mathrm{BC}$ is the first order ascending curve. Its description is then given as

$$
\begin{gathered}
f_{+}=\tanh \left(x-a_{0}\right)+b_{0}+c_{1 u} \frac{\tanh \left(x_{m}-a_{0}\right)-\tanh \left(x-a_{0}\right)}{\tanh \left(x_{m}-a_{0}\right)-\tanh \left(x_{r}-a_{0}\right)}, \\
c_{1 u}=\tanh \left(x_{r}+a_{0}\right)-\tanh \left(x_{r}-a_{0}\right)-2 b_{0} .
\end{gathered}
$$

Similar patterns may be developed iteratively for $n$-th order curve using a $(n-1)$-th order curve. Any reversal point should lie on the anhysteretic curve. For the details the Readers are referred to Refs. [73, 74]. At this point it should be remarked that the knowledge of last reversal point makes it possible to predict the shape of successive reversal curve, thus this approach, similar to the previously discussed concept for the J-A model [13], is much more useful for electrical engineers dealing with transient states than the idea of "working fraction" presented in Figure 3. 
The description equivalent to the one given as (5) using tangible physical units is presented below. Parameter $a$ is responsible for loop shape (named after its counterpart in the J-A model).

$$
\begin{gathered}
M=M_{s} \tanh \left(\frac{H_{e f f} \mp H_{c}^{0}}{a}\right) \pm b_{1}\left(H_{T I P}, M_{T I P}\right) \\
b_{1}\left(H_{T I P}, M_{T I P}\right)=0.5 M_{s}\left[\tanh \left(\frac{H_{e f f}^{T I P}+H_{c}^{0}}{a}\right)-\tanh \left(\frac{H_{e f f}^{T I P}-H_{c}^{0}}{a}\right)\right] \\
H_{e f f} \approx H+\alpha M .
\end{gathered}
$$

The last equation defines the effective field for quasi-static magnetization conditions.

\section{Exemplary modelling}

Measurements of symmetric hysteresis loops have been carried out for a non-oriented steel grade M330-35A from Stalprodukt S. A $^{1}$ using a computer-aided setup and a Single Sheet Tester device at low excitation frequency $f \approx 5 \mathrm{~Hz}$. It was assumed that for that frequency the rate-dependent effects due to eddy currents could be neglected. The induction waveform was controlled to be sine during the tests. The magnetisation waveform could be also assumed sine, as in soft magnetic materials $M(t) \gg H(t)$. Fragments of measured hysteresis loops were used to mimick reversal curves, as these could not be measured using a setup, which fulfills all the requirements of international standards IEC 60404.

In order to grasp the idea how to model reversal curves, let us have a look at Figure 4. Magnetisation varies along the descending branch of hysteresis loop according to $M(t)=M_{s}$ $\cos \omega t=M_{s} \cos (2 \pi f t)$, whereas along the ascending curve: according to $M(t)=-M_{s}$ $\cos \omega t=-M_{s} \cos (2 \pi f t)$, where $t \in\langle 0 ; T / 2\rangle=\langle 0 ; 1 /(2 f)\rangle$ and $f$ is the excitation frequency.

Some years ago Zirka and Moroz have introduced the concept of "transplantation" of fragments of major loop branches in order to describe the reversal curves and minor loops [83]. In other words they have copied and offsetted some appropriate loop fragments. In Figure 4 a modification of their offsetting concept has been depicted, in the sense that at an arbitrary reversal instant $t_{0}$ the time scale for the input signal is offset by $T / 2$, i.e. the input signal of the reversal curve follows the waveform of the other loop branch from the instant $T / 2-t_{0}$. The output signal is calculated using a hysteresis model, e.g. the one proposed by Takács. The presented approach to offset the input signal in time domain is aimed at development of the full rate-dependent model in the forthcoming work. The rate dependent model shall incorporate not only the quasi-static $M=\hat{\Gamma}\left(H_{\text {eff }}\right)$ or $H_{\text {eff }}=\hat{\Gamma}^{-1}(M)$ dependence, but also a term dependent on time derivative of input signal.

\footnotetext{
${ }^{1}$ This grade is out of production, as the enterprise produces only high quality grain oriented steel at present.
} 


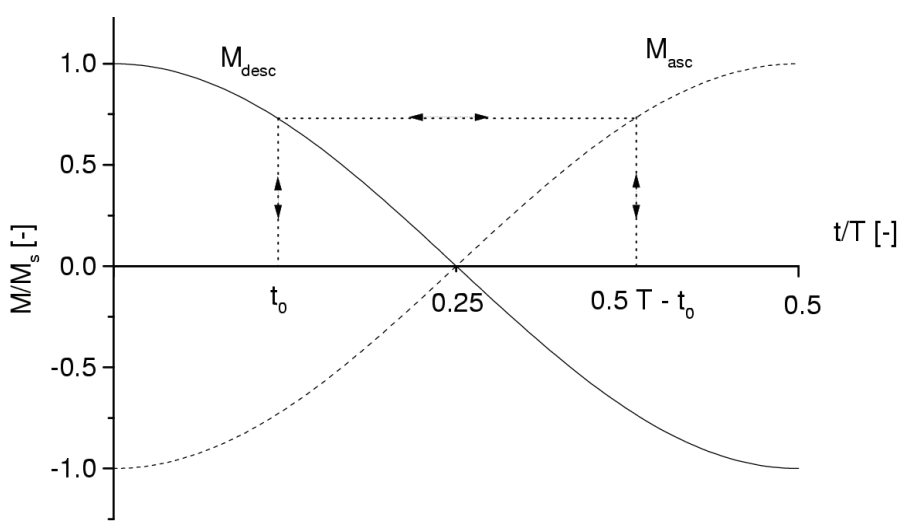

Fig. 4. "Transplantation" of input signal at reversal

In order to describe the reversal curve having magnetization as the input signal to the model, it is necessary to invert the relationship (6). Notation $T()=.\tanh ($.$) is used for brevity.$ At reversal point with $x=x_{r}$ the equality $T\left(x_{r}+a_{0}\right)-b_{0}=T\left(x_{r}-a_{0}\right)+b_{0}+c_{1 u}$ follows. For any further point belonging to the ascending reversal curve the relationship (6) is valid

$$
\begin{aligned}
& f_{+}\left[T\left(x_{m}-a_{0}\right)-T\left(x_{r}-a_{0}\right)\right]=T\left(x-a_{0}\right)\left[T\left(x_{m}-a_{0}\right)-T\left(x_{r}-a_{0}\right)\right]+\ldots \\
& +b_{0}\left[T\left(x_{m}-a_{0}\right)-T\left(x_{r}-a_{0}\right)\right]+c_{1 u} T\left(x_{m}-a_{0}\right)-c_{1 u} T\left(x-a_{0}\right),
\end{aligned}
$$

thus

$$
T\left(x-a_{0}\right)=\frac{\left(f_{+}-b_{0}\right)\left[T\left(x_{m}-a_{0}\right)-T\left(x_{r}-a_{0}\right)\right]-c_{1 u} T\left(x_{m}-a_{0}\right)}{T\left(x_{m}-a_{0}\right)-T\left(x_{r}-a_{0}\right)-c_{1 u}} .
$$

By applying the $T^{-1}()=.\operatorname{arc} \tanh ($.$) transformation to the last relationship one can calcu-$ late the sought $x$ values.

Dataset concerning a major loop measured at $B_{m}=1.5 \mathrm{~T}$ was used to recover model parameters. During the estimation process the sum of squared errors between the measured and the modelled loops was minimized. The obtained set of model parameters was: $\alpha=3 \cdot 10^{-5}[-]$, $\alpha=43.3[\mathrm{~A} / \mathrm{m}], H_{c}=44.8[\mathrm{~A} / \mathrm{m}]\left(a_{0}=1.34[-]\right), M_{s} \approx 1.19 \cdot 10^{6}[\mathrm{~A} / \mathrm{m}]$. The calculated value of $b_{0}=1.03 \cdot 10^{-7} \approx 0$ in dimensionless units.

Figure 5 depicts some examples of modelled higher order curves and measured symmetrical hysteresis loops. It can be stated that the shapes of respective fragments of corresponding magnetization curves are in a reasonable agreement. Therefore, a conclusion may be drawn that the Takács description, based on hyperbolic tangent mapping between magnetization and the effective field, may be a useful modelling tool for prediction of complex magnetization cycles.

However there exist some discrepancies between the predicted curves and experimental data. These may be due to:

- stucking of the procedure applied for estimation of model parameters in a local minimum (the obtained set of model parameters might be close to optimal one, but it does not neces- 
sarily yield the global minimum) - in the example depicted in Figure 5 the sum of squared errors calculated for 27 points belonging to the descending branch of the major loop was $9.82 \cdot 10^{10}\left[(\mathrm{~A} / \mathrm{m})^{2}\right]$;

- neglection of the reversible process in the description;

- accumulation of small errors in the calculations of successive curves;

- neglection of rate dependent effects in the description. In fact the shape of measured hysteresis loops may be affected by the effect of eddy currents even for low excitation frequency. This aspect of modelling is discussed in some detail in the successive section.

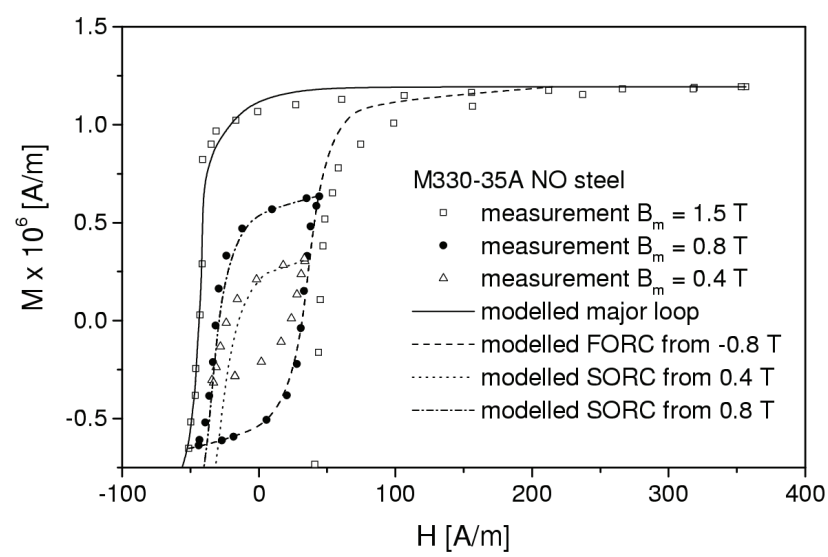

Fig. 5. Some modelled higher order reversal curves for a NO steel sheet; FORC denotes a First Order Reversal Curve (starting from a point on the major loop), whereas SORC stands for a Second Order Reversal Curve

\section{Effect of eddy currents}

It should be noted that hysteresis is generally speaking a rate-dependent phenomenon $[17,18]$. For conductive materials in the industrial frequency range of interest the dynamic effects are due mainly from eddy currents induced in the core material. In the case of electrical steels, however, one can distinguish a quasi-static regime without a notable hysteresis dependence on frequency [42]. It is beyond the scope of the present paper to resolve whether this phenomenon is due to frequency dependence of the microscopic dissipation mechanism and scaling invariance of dynamical equations, as suggested in [66], or it simply follows from the insufficient resolution of measurement devices [40].

The necessity to take into account in the J-A model the mutual interactions between hysteresis and eddy currents has been noticed and addressed by several authors [3, 10, 15, 22-24, $43,50,51,65,69,72,80]$.

Some of the aforementioned papers $[15,22,50,51,65,85]$ considered a combination of JA model with the statistical loss theory [8], which is regarded in the scientific community as one of the most advanced and sophisticated contemporary loss theories. Due to intrinsic 
limitations or inconsistencies of both theories (drawbacks of Bertotti's theory are revealed e.g. in $[11,25,31]$ the hybrid approach is not a perfect solution to the problem. Papers [20, 23] considered another approach, where total loss was separated into just two terms, the first one related to quasi-static hysteresis and the second one - to eddy currents induced in a wide spectrum of time- and spatial scales. The modification of shape of magnetization curves obtained as the result of the introduction of a fractional term due to eddy currents was achieved in a certain sense post hoc, the eddy currents were treated as a disturbance factor for the quasistatic loop. The rationale for loss separation into just two terms (and, consequently, denial of "excess loss" existence) was the Poynting theorem, which has just two terms if one neglects the displacement currents [82].

Yet another possibility to take into account eddy currents in hysteresis modelling follows from a direct inclusion of an appropriate term in the description of effective field. This approach seems particularly suited for circuit-oriented modelling $[1,2]$. Such approach was attempted for the J-A model for the first time in [72], where the influence of eddy currents was accounted with a reaction field (counterfield) term, calculated using the method of successive reactions. The author considered the $H$-excitation case for the geometry of cylinder-shaped core wound of extremely thin ribbons (specific to current transformers), what allowed him to make a number of approximations in the derivation of the final formula. His approach seems thus impossible to be extended to other geometries and types of excitation.

A somewhat similar approach has been proposed recently in [43], where the authors introduced into the effective field two additional terms of field strength related to classical and excess loss from Bertotti's theory. The aforementioned paper lacks, however, a discussion on the limitations of the proposed approach. In particular, it remains unclear how to consider the $V_{0}=f\left(B_{m}\right)$ dependence for minor loops and reversal curves, this aspect has been raised earlier in [20]. Moreover, only symmetrical minor loops are modelled.

An alternative approach might be to consider implicitly a term related to rate dependence of magnetization in the expression for the effective fild $[1,2,26]$. The validity of this approach has been demonstrated for a number of phenomenological hysteresis models [3]. Moreover, this approach seems particularly useful for consideration of hysteresis in finite element codes, provided the considered material is "soft" enough to neglect the difference between the $1 / \mu_{0} d B / d t$ and $d M / d t$ rates. A recent paper [26] suggested the following generalized expression for effective field:

$$
H_{\text {eff }}=H+\alpha M-\beta \operatorname{sign}\left(\frac{\mathrm{dM}}{\mathrm{dt}}\right)\left|\frac{\mathrm{dM}}{\mathrm{dt}}\right|^{v},
$$

where the (possibly fractional) exponent $v$ takes into account the effects from eddy currents generated in different time- and spatial scales within the magnetic core. Its value is influenced by a number of factors, e.g. the flow of inter-laminar eddy currents, mechanical strains of the wound core or (often neglected in the analysis) the capacitance coupling between adjacent ribbons [63].

Future work shall be aimed at an in-depth analysis of two possible approaches how to model the rate dependent effects in the Takács hysteresis model: 
- the first one, which assumes that there are two totally independent terms, related to hysteresis and eddy currents, respectively (this is justified by the existence of just two terms in the Poynting theorem), following the guidelines given in [20],

- the second one, which explicitly defines an additional term related to $d M / d t$ in the definition of the effective field $[3,26]$. The latter approach in fact denies the possibility to separate the loss components in the ferromagnetic material.

\section{Conclusions}

The paper has presented some ideas important in hysteresis modelling using the JilesAtherton model as a reference, due to its popularity in the scientific community. The merit of the Jiles-Atherton formalism lies in noticing the role of concepts of effective field and anhysteretic magnetization as important components of hysteresis descriptions. The model developers have also made a distinction between irreversible and reversible magnetization processes. The combination of different physical ideas allowed them to formulate a low dimensional description. Such modelling frameworks are of interest, e.g. to electrical engineers.

However, the Jiles-Atherton model lacks accuracy in representation of more complex magnetization cycles. Some of the reasons for this state of matter are explained in this paper. The existing model modifications aimed at patching of ad hoc noticed problems lead to a substantial complication of model equations, making it less attractive from the engineers' perspective. Some of the patches are only partially justified (introduction of $\delta_{M}$ term), moreover their very presence limits the range where the J-A model may be safely applied (as a consequence e.g. the original estimation procedure [49] fails to recover optimal values of model parameters). Moreover, some of J-A model parameters have to be modified for minor loops and/or reversal curves, but the developed pattern for their update requires the knowledge of both the previous and the next reversal points. The proposed modification is physically judged, but again makes the description less attractive to electrical engineers.

An accurate representation of hysteresis phenomenon should reproduce accurately not only major loops, but also more complicated reversal curves. Those may be subject to Madelung rules, which are recalled in the paper.

The phenomenological Takács description, based on a hyperbolic tangent mapping, appears to be an interesting alternative to the Jiles-Atherton model for engineering applications. Some of its advantages are rigorous mathematical foundations and flexibility. The variables involved in the mapping have been identified previously as magnetization and the effective field. Contrary to the Jiles-Atherton description there is no need to describe their relationship with an ordinary differential equation.

In order to demonstrate the usefulness of the Takács description, some higher order reversal curves have been modelled under the assumption that rate dependent effects could be neglected. The obtained curves have been compared to fragments of measured hysteresis loops. A reasonable agreement between the aforementioned curves has been obtained.

Finally some concepts for future work concerning the development of a rate dependent hysteresis model have been outlined. Two methods to include the rate dependent effects due 
mostly to eddy currents have been indicated. One of them relies on loss separation into two independent components, the other one introduces an additional term to the effective field, thus it "stitches" both components together. Future research shall compare the results obtained with both approaches, thus it should be useful to provide an answer to the non-trivial question raised during the last Soft Magnetic Materials conference in September 2011: "Is there any physical basis for loss separation?" [32]. The issue is important, because the possibility to separate loss in soft magnetic materials is taken for granted by the engineering community, moreover loss separation procedures appear explicitly in some standards concerning material characterization.

\section{Acknowledgements}

The work has been carried out within the framework of research grant N N510 702540 from National Research Centre. The author is grateful for an interesting exchange of views on the Jiles-Atherton model with Professors S.E. Zirka and R.G. Harrison. Stalprodukt S.A. is thanked for supplying steel samples. The author acknowledges granting access to measurement setup at Electrotechnical Institute, Wrocław.

\section{References}

[1] Andrei P., Caltun O. Stancu A1., Rate dependence of first-order reversal curves by using a dynamic Preisach model of hysteresis. Physica B, 372: 265-8 (2006).

[2] Andrei P., Stancu Al., Caltun O., Modeling and simulation of electrical circuits with hysteretic inductors by using a dynamic Preisach model. [In:] Preisach Memorial Book, Iványi A. (Ed.), Akádémiai Kiadó, Budapest, pp. 187-197 (2005).

[3] Andrei P., Stancu Al., Hauser H., Fulmek P., Temperature, stress, and rate dependent numerical implementation of magnetization processes in phenomenological models. Journal of Optoelectronics and Advanced Materials 9(4): 1137-1139 (2007).

[4] Atherton D.L., Schönbächler M., Measurements of reversible magnetization component. IEEE Transactions on Magnetics 24(1): 616-20 (1988).

[5] Azzaoui S., Srairi K., El Hachemi Benbouzid M., Non-linear magnetic hysteresis modelling by finite volume method for Jiles-Atherton model optimizing by a genetic algorithm. Journal of Electromagnetic Analysis and Applications 3: 191-198 (2011).

[6] Benda O., Barta Št., Open problems in the Preisach modeling of magnetic hysteresis. [In:] Nonlinear Electromagnetic Systems. A.J. Moses, A. Basak (Eds.), IOS Press, pp. 380-385, Amsterdam (1996).

[7] Benda O., Bydzovský J., Problems in modelling reversible processes in soft magnetic materials. Journal of Magnetism and Magnetic Materials 160: 87-88 (1996).

[8] Bertotti G., General properties of power losses in soft magnetic materials. IEEE Transactions on Magnetics 24(1): 621-630 (1988).

[9] Bertotti G., Connection between microstructure and magnetic properties of soft magnetic materials. Journal of Magnetism and Magnetic Materials 320: 2436-2442 (2008).

[10] Brockmeyer A., Schülting L., Modelling of dynamic losses in magnetic materials. Proceedings EPE'93, Brighton, UK, 13-16.09.1993, pp. 112-117 (1993).

[11] Broddefalk A., Lindenmo M., Dependence of the power losses of a non-oriented 3\% Si-steel on frequency and gauge. Journal of Magnetism and Magnetic Materials 320: e586-588 (2006).

[12] Calle C., Cuellar F., Guzmán O., Mendoza A., Simulaciones de comportamientos hysteréticos blandos en péliculas magnéticas. Revista Colombiana de Física 38: 541-544 (2006).

[13] Carpenter K.H., A differential equation approach to minor loops in the Jiles-Atherton model of hysteresis. IEEE Transactions on Magnetics 27(1): 4404-4406 (1991). 
[14] Carpentieri M., Azzerboni B., Finocchio G., La Foresta F., State-Independent Hypothesis to model the behaviour of magnetic materials. Journal of Magnetism and Magnetic Materials 280: 158-163 (2004).

[15] Chandrasena W., McLaren P.G., Annakage U.D. et al., Simulation of hysteresis and eddy current effects in a power transformer. Electric Power System Research 76(8): 634-641 (2006).

[16] Chiesa N., Power transformer modeling for inrush current calculation. PhD Thesis, Norwegian Technical University of Science and Technology, Trondheim (2010).

[17] Chua L.O., Stromsmoe K., Lumped-circuit models for nonlinear inductors exhibiting hysteresis loops. IEEE Transactions on Circuit Theory 17(4): 564-574 (1970).

[18] Chua L.O., Bass S.C., A generalized hysteresis model. IEEE Transactions on Circuit Theory 19(1): 36-48 (1971).

[19] Chwastek K., Szczygłowski J., An alternative method to estimate the parameters of Jiles-Atherton model. Journal of Magnetism and Magnetic Materials 314: 47-51 (2007).

[20] Chwastek K., Frequency behaviour of the modified Jiles-Atherton model. Physica B 403: 2484-2487 (2008).

[21] Chwastek K., Szczygłowski J., Estimation methods for the Jiles-Atherton model parameters - a review. Przegląd Elektrotechniczny 12: 145-148 (2008).

[22] Chwastek K., Modeling of dynamic hysteresis loops using the Jiles-Atherton approach. Mathematical and Computer Modelling of Dynamical Systems 15(1): 95-105 (2009).

[23] Chwastek K., Modelling offset minor hysteresis loops with the modified Jiles-Atherton description. Journal of Physics D: Applied Physics: 42: 16, pp. 165002 (2009).

[24] Chwastek K., Szczygłowski J., Wilczyński W., Modelling dynamic hysteresis loops in steel sheets. COMPEL 28(3): 603-612 (2009).

[25] Chwastek K., AC loss density component in electrical steel sheets. Philosophical Magazine Letters 90, 11: 809-817 (2010).

[26] Chwastek K., A dynamic extension to the Takács model. Physica B 405: 3800-3802 (2010).

[27] Chwastek K., Modelling magnetic properties of MnZn ferrites with the modified Jiles-Atherton description. Journal of Physics D: Applied Physics 43: 015005-5 (2010).

[28] Chwastek K., Szczygłowski J., Wilczyński W., Minor loops in the Harrison model. Acta Physica Polonica A 121: 941-944 (2012).

[29] Chwastek K., Szczygłowski J., The effect of anisotropy in the modified Jiles-Atherton model of static hysteresis. Archives of Electrical Engineering 60(1): 49-57 (2011).

[30] Deane J.H.B., Modelling the dynamics of nonlinear inductor circuits. IEEE Transactions on Magnetics 30(5): 2795-2801 (1994).

[31] de Campos M., Falleiros I.G.S., Landgraf F.J.G., Análise crítica do modelo das perdas em excesso. (Critical analysis of the model of excess losses. In Portuguese). Proceedings 58o Congresso Annual da ABM, Rio de Janeiro, 21-23.07.2003, pp. 2237-2246 (2003).

[32] de Campos M., Landgraf F.J.G., Is there any physical basis for the loss separation procedure? Soft Magnetic Materials Conference, Kos, Greece. (Poster S02-P0445) (2011).

[33] De Leon Fr., Semlyen A., A simple representation of dynamic hysteresis loops in power transformers. IEEE Transactions on Power Delivery 10(1): 315-321 (1995).

[34] Della Torre E., Oti J., Kádár Gy., Preisach modelling and reversible magnetization. IEEE Transactions on Magnetics 26(6): 3052-3058 (1990).

[35] Della Torre E., Magnetic hysteresis. IEEE Press, Piscataway (1999).

[36] Dlala E., Belahcen A., Arkkio A., Efficient magnetodynamic lamination model for two-dimensional field simulation of rotating electrical machines. Journal of Magnetism and Magnetic Materials 320(20): e1006-10 (2008). See also: Dlala E., Magnetodynamic vector hysteresis model for steel laminations of rotating electrical machines. PhD Thesis, Helsinki University of Technology, Espoo (2008).

[37] Dupré L., Melkebeek J.A.A., Electromagnetic hysteresis modelling: from material science to finite element analysis of devices. International Compumag Society Newsletter 10(3): 4-15 (2003).

[38] Dupré L., Sablik M.J., Van Keer R., Melkebeek J., Modelling of microstructural effects on magnetic hysteresis properties. Journal of Physics D: Applied Physics 35: 2086-2090 (2002).

[39] Faiz J., Saffari S., A new technique for modeling hysteresis in soft magnetic materials. Electromagnetics 30(4): 376-401 (2010). 
[40] Gozdur R., Wyznaczanie quasi-statycznej pętli histerezy blach elektrotechnicznych. (Determination of quasi-static hysteresis loop of electrical steel. In Polish), Przegląd Elektrotechniczny 2: 147-149 (2004).

[41] Gyselink J., Dular P., Sadowski N. et al., Incorporation of a Jiles-Atherton vector hysteresis model in 2D FE magnetic field computations. COMPEL 23(3): 685-693 (2004).

[42] Haller T.R., Kramer J.J., Observation of dynamic domain size variation in a silicon-iron alloy. Journal of Applied Physics 41(3): 1034-1035 (1970).

[43] Hamimid M., Mimoune S. M., Féliachi M., Hybrid magnetic formulation based on the losses separation method for modified dynamic inverse Jiles-Atherton model. Physica B 406: 2755-2757. (2011).

[44] Harrison R., A physical model of spin ferromagnetism. IEEE Transactions on Magnetics 39(2): 950960 (2003).

[45] Harrison R., Positive-feedback theory of hysteresis recoil loops in hard ferromagnetic materials. IEEE Transactions on Magnetics 47(1): 175-191 (2011).

[46] Jiles D.C., Atherton D.L., Theory of ferromagnetic hysteresis. Journal of Magnetism and Magnetic Materials 61: 48-60 (1986).

[47] Jiles D.C., A self-consistent generalized model for the calculation of minor loop excursions in the theory of hysteresis. IEEE Transactions on Magnetics 28(5): 2602-2604 (1992).

[48] Jiles D.C., Thoelke J.B., Theory of ferromagnetic hysteresis: determination of model parameters from experimental hysteresis loops. IEEE Transactions on Magnetics 25(5): 3928-3930 (1989).

[49] Jiles D.C., Thoelke J.B., Devine M.K., Numerical determination of hysteresis parameters for the modelling of magnetic properties using the theory of ferromagnetic hysteresis. IEEE Transactions on Magnetics 28(5): 27-35 (1992).

[50] Jiles D.C., Frequency dependence of hysteresis curves in conducting magnetic materials. Journal of Applied Physics 76(10): 5849-5855 (1994).

[51] Jiles D.C., Modelling the effect of eddy current losses on frequency dependent hysteresis in electrically conducting media. IEEE Transactions on Magnetics 30(6): 4326-4328 (1994).

[52] Kádár Gy., The bilinear product model of hysteretic phenomena. Physica Scripta T25, 161-164 (1989)

[53] Kádár Gy., Szabó Zs., Magnetization process as a combined function of field and temperature in the product model of hysteresis. Journal of Magnetism and Magnetic Materials, pp. 272-276, e547-9 (2004).

[54] Kádár Gy., The Preisach-type product model of magnetic hysteresis. (In:) A. Iványi (Ed.), Preisach Memorial Book, Akadémiai Kiadó, Budapest, pp. 15-27 (2005).

[55] Kleineberg T., A generalized approach for modelling the nonlocal memory of hysteretic systems. Journal of Magnetism and Magnetic Materials 166: 315-320 (1999).

[56] Knypiński Ł., Nowak L., Sujka P., Radziuk K., Application of a PSO algorithm for identification of the parameters of Jiles-Atherton hysteresis model. IET Conference Proceedings 2011(Issue CP577): 134-135, DOI: 10.1049/cp.2011.0070 (2011).

[57] Kobayashi S., Takahashi S., Shishido T. et al., Low field magnetic characterization of ferromagnets using a minor-loop scaling law. Journal of Applied Physics 107, 023908 (6 pp.) (2010).

[58] Lederer D., Igarashi H., Kost A., Honma T., On the parameter identification and application of the Jiles-Atherton hysteresis model for numerical modelling of measured characteristics. IEEE Transactions on Magnetics 35(3): 1211-1214 (1999).

[59] Łyskawiński W., Sujka P., Szelag W., Barański, M., Numerical analysis of hysteresis loss in pulse transformer. Archives of Electrical Engineering 60(2): 187-195 (2011).

[60] Madelung E.R., Über Magnetisierung durch schnellverlaufende Strome und die Wirkungsweise der Rutherford-Marconischen Magnetdetektors. Annalen der Physik 17(5): 861-863 (1905).

[61] Mayergoyz I.D., Mathematical models of hysteresis. Springer-Verlag, New York (1991).

[62] Mousavi S.A., Engdahl G., Differential approach of scalar hysteresis modeling based on the Preisach theory. IEEE Transactions on Magnetics 47: 3040-3043 (2011).

[63] Mukerji S.K., George M., Ramamurthy M.B., Asaduzzaman K., Eddy currents in laminated rectangular cores. Progress in Electromagnetics Research 83: 435-445 (2008). 
[64] Pierce M.S., Buechler C.R., Sorensen L.B. et al., Disorder-induced magnetic memory: Experiments and theories. Physical Review B 75(144406) (23 pp.). Also available at http: //arxiv.org/condmat/061142v2 (2007).

[65] Philips D.A., Dupré L.R., Melkebeek J.A.A., Comparison of Jiles and Preisach hysteresis models in magnetodynamics. IEEE Transactions on Magnetics 31(6): 3551-3553 (1995).

[66] Rao M., Frequency-independent hysteresis in metallic magnets. Physical Review B 45(13): 75297531 (1992).

[67] Rezaei-Zare A., Iravani R., On the transformer core dynamic behavior during electromagnetic transients. IEEE Transactions on Power Delivery 25(3): 1606-1619 (2010).

[68] Sablik M., Jiles D.C., Coupled magnetoelastic theory of magnetic and magnetostricitve hysteresis. IEEE Transactions on Magnetics 29(3): 2113-2123 (1993).

[69] Sadowski N., Batistela N., Bastos J.P.A., Lajoie-Mazenc M., An inverse Jiles-Atherton model to take into account hysteresis in time-stepping finite-element calculations. IEEE Transactions on Magnetics 38(2): 797-800 (2002).

[70] Schneider C.S., Winchell S.D., Hysteresis in conducting ferromagnets. Physica B 372: 269-272 (2006).

[71] Steinmetz C.P., On the law of hysteresis. AIEE Transactions, 3-64, reprinted under the title: A Steinmetz contribution to the ac power revolution. (Introd. J.E. Brittain) (1984), Proceedings of the IEEE 72(2): 197-221 (1892).

[72] Szczygłowski J., Influence of eddy currents on magnetic hysteresis loops in soft magnetic materials. Journal of Magnetism and Magnetic Materials 223: 97-102 (2001).

[73] Takács J., A phenomenological mathematical model of hysteresis. COMPEL 20(2): 1002-1014 (2001).

[74] Takács J., Mathematics of hysteretic phenomena. Wiley-VCH, Weinheim (2003).

[75] Takahashi S., Zhang L., Minor hysteresis loop in Fe metal and alloys. Journal of the Physical Society of Japan 73(6): 1567-1575 (2004).

[76] Takahashi S., Kobayashi S., Kamada Y. et al., Analysis of minor hysteresis loops and dislocations in Fe., Physica B 372: 190-193 (2006).

[77] Takahashi S., Kobayashi S., Scaling power-law relations in asymmetrical minor hysteresis loops. Journal of Applied Physics 107, 063903 (5 pp.) (2010).

[78] Talukdar S.N., Bailey J.R., Hysteresis models for system studies. IEEE Transactions on Power Apparatus and Systems 95: 1429-1434 (1976).

[79] Toms H.L., Colclaser R.G., Krefta M.P., Two-dimensional finite element magnetic modelling for scalar hysteresis effects. IEEE Transactions on Magnetics 37(2): 982-988 (2001).

[80] Theocharis A.D., Milias-Argitis J., Zacharias Th. (2008), Single-phase transformer model including magnetic hysteresis and eddy currents. Electrical Engineering 90: 229-241.

[81] Weiss P., L'hypothèse du champ moléculaire et la propriété ferromagnétique. Journal de Physique 4(6): 665-690 (1907).

[82] Young F.J., On the validity of characterizing eddy current phenomena by parabolic partial differential equations. IEEE Transactions on Magnetics 14(6): 1185-1186 (1978).

[83] Zirka S.E., Moroz Yu.I., Hysteresis modeling based on transplantation. IEEE Transactions on Magnetics 31(6): 3509-3511 (1995).

[84] Zirka S.E., Moroz Yu.I., Hysteresis modeling based on similarity. IEEE Transactions on Magnetics 35(4): 2090-2096 (1999)

Further information on the Jiles-Atherton description and some aspects of its dynamic extension may be found in:

[85] Zirka S.E., Moroz Yu.I., Harrison R.H., Chwastek K., On physical aspects of the Jiles-Atherton hysteresis model. Journal of Applied Physics 112: 043916 (2012). 